\title{
Diet patterns among low income women in Mumbai
}

\author{
Sarah Kehoe ${ }^{1}$, Devi Shivashankaran ${ }^{2}$, Harsha Chopra $^{2}$, Sirazul Sahariah ${ }^{2}$, Meera Gandhi ${ }^{2}$, \\ Nicola Winder ${ }^{1}$, Barrie Margetts ${ }^{3}$, Ramesh Potdar ${ }^{2}$ and Caroline Fall ${ }^{1}$ \\ ${ }^{1}$ MRC Lifecourse Epidemiology Unit, Southampton, SO16 6YD. ${ }^{2}$ Centre for Study of Social Change, Mumbai, India and \\ ${ }^{3}$ Public Health Nutrition, University of Southampton, SO16 6YD, UK
}

Poor nutritional status of young women before and during pregnancy can result in sub-optimal foetal development. In 2005-6, approximately a quarter of women living in Mumbai slums were chronically undernourished (BMI $<18.5 \mathrm{~kg} / \mathrm{m}^{2}$ ) and $46 \%$ had iron deficiency anaemia $(\mathrm{Hb}<12 \mathrm{~g} / \mathrm{dL})^{(1)}$. There is evidence of low intakes of micronutrient-rich foods among women of reproductive age living in Mumbai slums ${ }^{(2)}$, yet little is known about dietary patterns in this population. Our objectives were to identify diet patterns of low income slum-dwelling women and to study associations with socio-demographic factors and BMI. Participants were 4816 women aged $25 \pm 4$ years registered in the Mumbai Maternal Nutrition Project (MMNP). We collected demographic and anthropometric data from the women at registration. A trained project assistant administered a 212 item food frequency questionnaire (FFQ) with a reference period of the past week. The FFQ foods were condensed to 40 food groups and principal component analysis of the food group variables was conducted. We used univariate and multivariate regression models to study associations between pattern scores, BMI and demographic variables.

The women were light and thin. Median (IQR) BMI was $20.0(17.9,22.9) \mathrm{kg} / \mathrm{m}^{2}$. The diet pattern that accounted for most variance $(8.7 \%)$ was characterised by frequent intakes of snacks, fruit, green leafy vegetable curry and desserts ('snack and fruit' pattern). The second pattern explained $5.3 \%$ of the variance and was characterised by frequent intakes of biriyani and mutton and low intakes of legumes and coconut ('non-vegetarian'). The third pattern was named 'fish and coconut' as it was characterised by frequent intakes of these foods. Adherence to the 'snack and fruit' pattern was positively associated with skill level of occupation $(p=0.001)$ and standard of living $(p=0.003)$. Adherence to the non-vegetarian pattern was associated with younger age, being Muslim, lower education status and lower standard of living. The 'fish and coconut' pattern was positively associated with education and SLI score $(p<0.001)$. BMI was positively associated with snack and fruit and non-vegetarian scores $(p<0.01)$. There was a negative association between rice and coconut scores and BMI but this was not statistically significant $(p=0.118)$.

This study provides new information on diet patterns among low income Indian women and shows that these patterns are related to demographic variables and nutritional status. This information can be used to target dietary interventions to at-risk-groups more effectively and may be useful in developing food-based dietary guidelines for this population.

1. Arnold F, Parasuraman S, Arokiasamy P \& Kothari M (2009) Nutrition in India. National Family Health Survey (NFHS-3), India, 2005-06. Mumbai: International Institute for Population Sciences.

2. International Institute for Population Sciences (2007) National Family Health Survey (NFHS-3) 2005-06 Volume 1. Mumbai. 